\title{
CONCERNING NON-MEASURABLE SUBSETS \\ OF A GIVEN MEASURABLE SET
}

H. W. PU

(Received 12 December 1969)

Communicated by B. Mond

Let $R, \mu$ and $M_{\mu}$ denote the set of real numbers, Lebesgue outer measure and the class of Lebesgue measurable subsets of $R$ respectively. It is easy to prove that the complement $E^{c}$ of $E \in M_{\mu}$ is a set of Lebesgue measure zero if the inequality $\mu(E \cap I) \geqq \delta \mu(I)$ holds for some $\delta>0$ and all intervals $I$ of $R$. However, in [1], Hewitt raised a problem whether the result is still true if $E$ is not a priori measurable set. In this paper, a negative answer to this question is given through a counter-example. Also, it is proved that for a given set $E \in M_{\mu}$ with $\mu(E)>0$ there is a non-measurable subset $A$ of $E$ satisfying $\mu(A)=\mu(E)$.

Lemma 1. Let $E \in M_{\mu}$ with $\mu(E)<\infty$ and $A \subset E$. Then $A \in M_{\mu}$ if and only if $\mu(E)=\mu(A)+\mu(E-A)$.

For the proof, the reader is referred to [2].

LemMa 2. If $\left\{E_{i}\right\}$ is a sequence of pairwise disjoint sets of $M_{\mu}$ each having positive measure and $\left\{A_{i}\right\}$ is a sequence of non-measurable sets such that $A_{i} \subset E_{i}$ for each $i$, then $\bigcup_{i=1}^{\infty} A_{i}$ is non-measurable and $\mu\left(\bigcup_{i=1}^{\infty} A_{i}\right)=\sum_{i=1}^{\infty} \mu\left(A_{i}\right)$.

PROOF. The non-measurability for $\bigcup_{i=1}^{\infty} A_{i}$ is obvious. We need only prove

$$
\mu\left(\bigcup_{i=1}^{\infty} A_{i}\right) \geqq \sum_{i=1}^{n} \mu\left(A_{i}\right)
$$

for every $n$, from which $\mu\left(\bigcup_{i=1}^{\infty} A_{i}\right) \geqq \sum_{i=1}^{\infty} \mu\left(A_{i}\right)$ follows, and the conclusion is obtained in view of subadditivity of $\mu$. By monotoneity of $\mu$,

$$
\mu\left(\bigcup_{i=1}^{\infty} A_{i}\right) \geqq \mu\left(\bigcup_{i=1}^{n} A_{i}\right)
$$

for all $n$. We shall show that $\mu\left(\bigcup_{i=1}^{n} A_{i}\right)=\sum_{i=1}^{n} \mu\left(A_{i}\right)$ by induction. The equality is trivial for $n=1$. Assume that it holds for $n=k$. Since $A_{k+1} \subset E_{k+1}$ and $\bigcup_{i=1}^{k} A_{i} \subset E_{k+1}^{c}$, measurability of $E_{k+1}$ implies that

$$
\mu\left(\bigcup_{i=1}^{k+1} A_{i}\right)=\mu\left(\bigcup_{i=1}^{k} A_{i}\right)+\mu\left(A_{k+1}\right)=\sum_{i=1}^{k+1} \mu\left(A_{i}\right)
$$

The last equality follows by inductive hypothesis. The proof is now completed. 
Lemma 3. If $E \in M_{\mu}$ with $\mu(E)>0$, then there is a non-measurable subset $A$ of $E$ such that $\mu(A) \geqq \frac{1}{2} \mu(E)$.

Proof. The existence of a non-measurable subset $Q$ of $E$ is well-known.

If $0<\mu(E)<\infty$, then by lemma $1, \mu(E)<\mu(Q)+\mu(E-Q)$. Thus we have $\mu(Q)>\frac{1}{2} \mu(E)$ or $\mu(E-Q)>\frac{1}{2} \mu(E)$. Since $Q \notin M_{\mu}, E-Q \notin M_{\mu}$. The conclusion follows.

If $\mu(E)=\infty$, then by $\sigma$-finiteness of $\mu$, there is a sequence of pairwise disjoint sets $\left\{E_{i}\right\}$ of $M_{\mu}$ such that $E=\bigcup_{i=1}^{\infty} E_{i}$ and $\mu\left(E_{i}\right)<\infty$ for each $i$ (we may assume $\left.0<\mu\left(E_{i}\right)<\infty\right)$. By what we have just shown, there is a non-measurable subset $A_{i}$ of $E_{i}$ for each $i$ such that $\mu\left(A_{i}\right)>\frac{1}{2} \mu\left(E_{i}\right)$. Let $A=\bigcup_{i=1}^{\infty} A_{i}$. By lemma 2, $A \notin M_{\mu}$ and

$$
\mu(A)=\sum_{i=1}^{\infty} \mu\left(A_{i}\right) \geqq \frac{1}{2} \sum_{i=1}^{\infty} \mu\left(E_{i}\right)=\frac{1}{2} \mu(E) .
$$

THEOREM. If $E \in M_{\mu}$ with $\mu(E)>0$, then there is a non-measurable subset $A$ of $E$ such that $\mu(A)=\mu(E)$.

Proof. Case 1. $0<\mu(E)<\infty$. We define $r_{0}=\mu(E)$ and $B_{0}=\emptyset$.

By lemma 3, there is $A_{1} \subset E$ such that $A_{1} \notin M_{\mu}$ and $\mu\left(A_{1}\right) \geqq r_{0} / 2$. Also, there is $B_{1} \in M_{\mu}$ such that $E-B_{0} \supset B_{1} \supset A_{1}$ and $\mu\left(B_{1}\right)=\mu\left(A_{1}\right)$. Let $r_{1}=$ $\mu\left(E-B_{1}\right)$. Clearly $0 \leqq r_{1} \leqq r_{0} / 2$.

If $r_{1}=0$, then we are through. Assume $r_{1}>0$. By the same reason, there are $A_{2} \notin M_{\mu}$ and $B_{2} \in M_{\mu}$ such that $A_{2} \subset B_{2} \subset E-\bigcup_{k=0}^{1} B_{k}$ and $\mu\left(B_{2}\right)=\mu\left(A_{2}\right) \geqq$ $r_{1} / 2$. Let $r_{2}=\mu\left(E-\bigcup_{k=0}^{2} B_{k}\right)$, then $0 \leqq r_{2} \leqq r_{1} / 2 \leqq r_{0} / 2^{2}$.

Suppose $\left\{A_{j}\right\}_{j=1}^{n},\left\{B_{j}\right\}_{j=0}^{n}$ and $\left\{r_{j}\right\}_{j=0}^{n}$ have been defined such that $A_{j} \notin M_{\mu}$, $B_{j} \in M_{\mu}, A_{j} \subset B_{j} \subset E-\bigcup_{k=0}^{j-1} B_{k}$,

$$
\mu\left(B_{j}\right)=\mu\left(A_{j}\right) \geqq r_{j-1} / 2, r_{j}=\mu\left(E-\bigcup_{k=0}^{j} B_{k}\right) \leqq r_{0} / 2^{j} \text { for } j=1,2, \cdots n .
$$

Clearly $\left\{B_{j}\right\}_{j=1}^{n}$ is pairwise disjoint. By lemma $2, \bigcup_{j=1}^{n} A_{j} \notin M_{\mu}$ and

$$
\mu\left(\bigcup_{j=1}^{n} A_{j}\right)=\sum_{j=1}^{n} \mu\left(A_{j}\right)=\sum_{j=1}^{n} \mu\left(B_{j}\right)=\mu\left(\bigcup_{j=1}^{n} B_{j}\right)=\mu(E)-r_{n} .
$$

If $r_{n}=0$, we may take $A=\bigcup_{j=1}^{n} A_{j}$. Otherwise, $\mu\left(E-\bigcup_{j=0}^{n} B_{j}\right)=r_{n}>0$ and there are $A_{n+1} \notin M_{\mu}, B_{n+1} \in M_{\mu}$ such that $A_{n+1} \subset B_{n+1} \subset E-\bigcup_{k=0}^{n} B_{k}$,

$$
\mu\left(B_{n+1}\right)=\mu\left(A_{n+1}\right) \geqq r_{n} / 2, r_{n+1}=\mu\left(E-\bigcup_{k=0}^{n+1} B_{k}\right) \leqq r_{0} / 2^{n+1}
$$

If this process does not terminate, we obtain infinite sequences $\left\{A_{i}\right\},\left\{B_{i}\right\}$ and $\left\{r_{i}\right\}$. Let $A=\bigcup_{i=1}^{\infty} A_{i}$. By lemma 2 again, $A \notin M_{\mu}$ and

$$
\mu(A)=\sum_{i=1}^{\infty} \mu\left(A_{i}\right)=\sum_{i=1}^{\infty} \mu\left(B_{i}\right)=\mu\left(\bigcup_{i=1}^{\infty} B_{i}\right)
$$


Thus

$$
\mu(E) \geqq \mu(A) \geqq \mu\left(\bigcup_{i=1}^{n} B_{i}\right)=\mu(E)-r_{n} \geqq \mu(E)\left(1-1 / 2^{n}\right)
$$

for all $n$. It follows that $\mu(A)=\mu(E)$.

Case 2. $\mu(E)=\infty$. By $\sigma$-finiteness of $\mu$, there is a sequence of pairwise disjoint sets $\left\{E_{i}\right\}$ of $M_{\mu}$ such that $E=\cup E_{i}, 0<\mu\left(E_{i}\right)<\infty$ for each $i$. The conclusion follows easily from case 1 and lemma 2 .

Finally, we proceed to the construction of a counter-example. Let $E=[0,1]$. By the above theorem, there is a $Q \subset E$ such that $Q \notin M_{\mu}$ and $\mu(Q)=\mu(E)=1$. Let

$$
A=(-\infty, 0) \cup Q \cup(1, \infty) .
$$

Obviously $A \notin M_{\mu}$, and therefore $\mu\left(A^{c}\right) \neq 0$. We assert that $\mu(A \cap I)=\mu(I)$ for every interval $I$ of $R$.

Case 1. $I \subset[0,1]$.

1.1. $0 \in I$ or $1 \in I$ : There is a subinterval $J$ of $[0,1]$ such that $I \cap J=\emptyset$, $I \cup J=[0,1]$, where $J$ may be empty or a singleton. Thus

$$
1=\mu(Q)=\mu(Q \cap I)+\mu\left(Q \cap I^{c}\right)=\mu(Q \cap I)+\mu(Q \cap J) .
$$

If $\mu(Q \cap I)<\mu(I)$, then we would have

$$
1=\mu(Q)=\mu(Q \cap I)+\mu(Q \cap J)<\mu(I)+\mu(J)=1 .
$$

This leads to a contradiction. Thus $\mu(Q \cap I)=\mu(I)$ and hence $\mu(A \cap I)=$ $\mu(Q \cap I)=\mu(I)$.

1.2. $0 \notin I$ and $1 \notin I$ : There are two subintervals $J_{1}, J_{2}$ of $[0,1]$ such that $J_{1}$, $I, J_{2}$ are pairwise disjoint and $J_{1} \cup I \cup J_{2}=[0,1]$. Since $J_{1} \cup I \in M_{\mu}, J_{1} \in M_{\mu}$, we have

$$
\begin{aligned}
1 & =\mu(Q)=\mu\left(Q \cap\left(J_{1} \cup I\right)\right)+\mu\left(Q \cap J_{2}\right) \\
& =\mu\left(Q \cap J_{1}\right)+\mu(Q \cap I)+\mu\left(Q \cap J_{2}\right) .
\end{aligned}
$$

If $\mu(Q \cap I)<\mu(I)$, then we would have

$$
1=\mu(Q)<\mu\left(J_{1}\right)+\mu(I)+\mu\left(J_{2}\right)=1 .
$$

This leads to a contradiction too. Thus $\mu(A \cap I)=\mu(Q \cap I)=\mu(I)$.

Case 2. $I \notin[0,1]$. Let $I_{1}=I \cap(-\infty, 0), I_{2}=I \cap[0,1]$ and $I_{3}=I \cap$ $(1, \infty)$ (some of them may be empty). Since $I_{1}, I_{2} \in M_{\mu}$,

$$
\begin{aligned}
\mu(A \cap I) & =\mu\left(A \cap I_{1}\right)+\mu\left(A \cap\left(I_{2} \cup I_{3}\right)\right) \\
& =\mu\left(A \cap I_{1}\right)+\mu\left(A \cap I_{2}\right)+\mu\left(A \cap I_{3}\right) \\
& =\mu\left(I_{1}\right)+\mu\left(A \cap I_{2}\right)+\mu\left(I_{3}\right) .
\end{aligned}
$$


By case 1, we have

$$
\mu(A \cap I)=\mu\left(I_{1}\right)+\mu\left(A \cap I_{2}\right)+\mu\left(I_{3}\right)=\mu\left(I_{1}\right)+\mu\left(I_{2}\right)+\mu\left(I_{3}\right)=\mu(I) .
$$

\section{References}

[1] E. Hewitt and K. Stromberg, Real and Abstract Analysis (Springer-Verlag, Berlin, 1965, p. 295).

[2] M. E. Munroe, Introduction to Measure and Integration (Addison-Wesley, Reading, Massachusetts, 1959, pp. 96-97).

Texas A \& $\mathbf{M}$ University

College Station, Texas 